EPJ Web of Conferences 61, 08009 (2013)

DOI: $10.1051 /$ epjconf/ 20136108009

(C) Owned by the authors, published by EDP Sciences, 2013

\title{
A Three-decade X-band VLBI Study of 3CR Lobe-dominated Quasar Nuclei
}

\author{
David H. Hough ${ }^{1, a}$ \\ ${ }^{1}$ Dept. of Physics \& Astronomy, Trinity University, San Antonio, TX 78212-7200 USA
}

\begin{abstract}
We report X-band VLBI observations of several 3CR lobe-dominated quasar nuclei from 1981 to 2010, mostly obtained with the NRAO VLBA. The goal is to follow flux density outbursts and to fully determine the jet morphology and kinematics on 1-100 pc scales. In 3C207, the core region has flux outbursts at mean intervals of $\sim 7 \mathrm{yr}$; one of these is actually a double outburst from a stationary true core and a swinging component $\sim 0.5$ mas apart. The position angle $(P A)$ of the swinging component varies by $\sim 40^{\circ}$, while the $P A$ values of the jet components span $\sim 25^{\circ}$. The jet extends to $\sim 25$ mas. Average superluminal speeds are $\sim 10 \mathrm{c}$. One component shows apparent acceleration from $7 c$ to $14 c$ at 2-3 mas from the true core, in a jet recollimation zone that redirects the flow toward $P A \sim 90^{\circ}$. Individual jet components expand until reaching the recollimation zone. In 3C263 and other objects, some of the same phenomena are seen, including ejection of jet components over a range in $P A$, superluminal motion, and apparent acceleration, but to a lesser degree. Possible physical interpretations involving beaming, orientation, projection, precession, and magnetic effects are discussed.
\end{abstract}

\section{Introduction}

We are conducting a long-term VLBI survey to test models of AGN jets and AGN unification [1]. In contrast to the many highly productive surveys of bright, compact AGN, e.g., [2, 3], we have selected a complete sample of 25 lobedominated quasars (LDQs) from the revised 3CR catalog [4]. Unlike the bright, compact AGN, the LDQs have been selected by their extended steep-spectrum radio emission, which minimizes orientation bias due to highly-beamed structures and permits tests of jet models over a wide range of orientations. The 3CR LDQ nuclei have flux densities that span a vast range, from $\sim 1 \mathrm{mJy}$ and $\sim 1 \mathrm{Jy}$.

The key results from our previous VLBI studies include: (1) All 25 LDQ nuclei are detected; (2) 22 have one-sided jets (3 have just faint cores); (3) the inner jets typically bend a few degrees; (4) jet component speeds in 15 sources range from 0 to at least $\sim 10 c ;(5)$ there is some evidence that outer components are faster than inner ones, but thus far only one component in one source has been followed long enough to actually observe its acceleration; (6) four sources have low-polarization core/inner jet regions with significant rotation measure, two of which have long ( 25-40 mas) moderately-polarized jets with longitudinal magnetic fields, e.g., [5-8].

We report here on a three-decade X-band VLBI study of six LDQ nuclei: 3C207, 3C208, 3C212, 3C245, $3 \mathrm{C} 249.1$, and 3C263. Observations were made at 10.7 $\mathrm{GHz}$ from 1981.76 to 1991.15 , and at $8.4 \mathrm{GHz}$ from 1991.16 to 2010.81 . These are the best-studied LDQ nuclei in the $3 \mathrm{CR}$ complete sample, and thus provide the best opportunity for determining the phenomenology in LDQ

\footnotetext{
ae-mail: dhough@trinity.edu
}

jets and its implications for jet models. While we do still find cases in which jet speeds are faster further from the core, the picture is more complex now, with stationary and swinging components, variable direction of component ejection, both acceleration and deceleration, and recollimation on 1-100 pc scales.

\section{The LDQ 3C207}

The LDQ 3C207 has the brightest VLBI core in our sample (up to $\sim 1.5 \mathrm{Jy}$ ), so its jet is probably oriented closest to our line-of-sight. VLBI observations from 1981 to 2010 have followed several flux density outbursts and revealed some very interesting jet morphology and kinematics on 1$100 \mathrm{pc}$ scales. The overall VLBI jet has weak features as far as $\sim 25$ mas to the east of the bright core region (Fig. 1). We have followed jet component evolution in the inner $\sim 6$ mas closest to the core region (Fig. 2). Modelfitting of the core region shows that it has a true core $\mathrm{C}$ and a swinging component $\mathrm{S} \sim 0.5$ mas east of $\mathrm{C}$ that exhibits little, if any, radial motion. The core region has shown dramatic variability by a factor of $\sim 5$. It has experienced four flux events/outbursts, at mean intervals of $\sim 7 \mathrm{yr}$ and peaking around 1983, 1988, 1995, and 2003, with a fifth beginning around 2010 (Fig. 3). Note that the 2003 outburst was actually a double outburst, with two events $\sim 2 \mathrm{yr}$ apart in the true core $\mathrm{C}$, each of which led to a delayed flare in the swinging component $\mathrm{S} \sim 1$ yr later. The position angle $(P A)$ of the swinging component $\mathrm{S}$ shows a general trend of increasing by $\sim 40^{\circ}$ over $\sim 25 \mathrm{yr}$, with a noteworthy backtrack of $\sim 10^{\circ}$ during the 2003 double outburst (Fig. 4). 


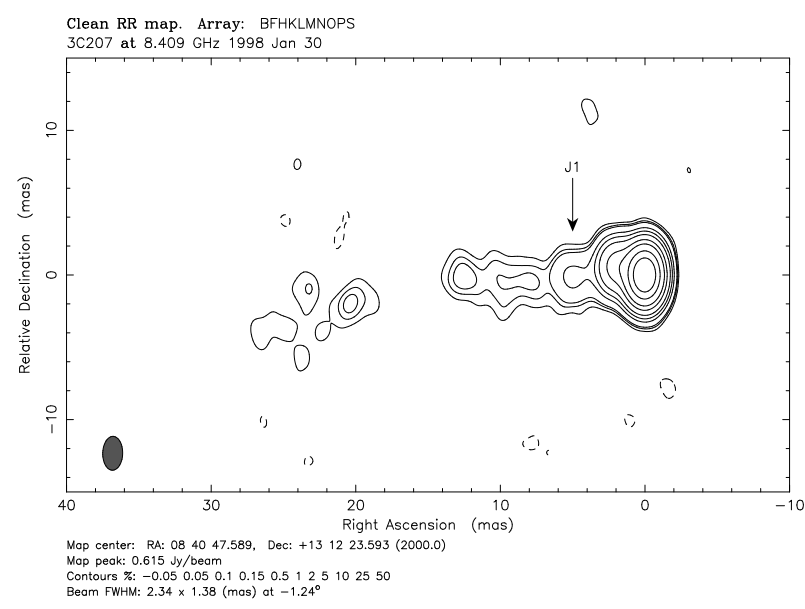

Figure 1. $8.4 \mathrm{GHz}$ VLBA image of overall 3C207 jet at epoch 1998.08. The jet component $\mathrm{J} 1$ is labeled.

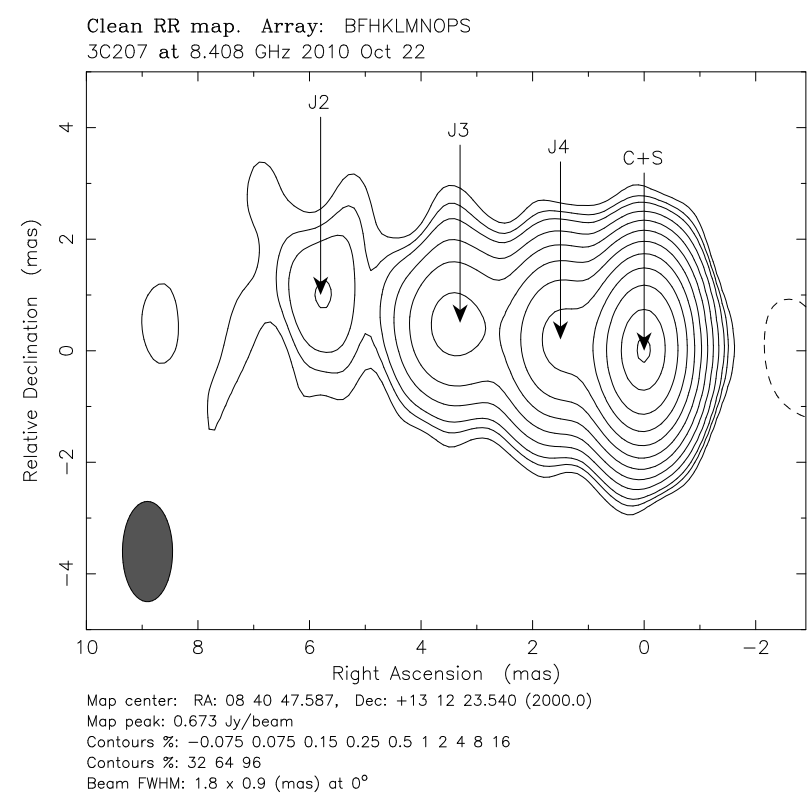

Figure 2. $8.4 \mathrm{GHz}$ VLBA image of inner $3 \mathrm{C} 207$ jet at epoch 2010.81. The true core $\mathrm{C}$, swinging component $\mathrm{S}$, and jet components $\mathrm{J} 2, \mathrm{~J} 3$, and $\mathrm{J} 4$ are labeled.

A plot of radial separation $R$ from the true core $\mathrm{C}$ vs. time for the swinging component $\mathrm{S}$ and the jet components J1, J2, J3, and J4 shows that all four jet components trace back reasonably well to the 1983, 1988, 1995, and 2003 outbursts (Fig. 5). A linear fit for component $\mathrm{S}$ shows a small, marginal outward motion over $30 \mathrm{yr}$ at $(0.3 \pm 0.1) c$. Linear fits for the four jet components yield superluminal speeds of $(7.8 \pm 1.9) c,(9.9 \pm 0.5) c,(9.7 \pm 0.3) c$, and $(3.0 \pm 0.7) c$, respectively, assuming standard cosmology. However, J2 shows apparent acceleration; J3 shows a hint of deceleration. Modeling the $\mathrm{J} 2$ acceleration as piecewise linear shows an increase from $7 c$ to $14 c$ at 2-3 mas from the true core (Fig. 6). Close to the core, where the $\mathrm{J} 2$ and $\mathrm{J} 4$ components are really doubles, we plot their brightness centroids. We have also examined the jet component trajectories on the sky (Fig. 7). The true core is at

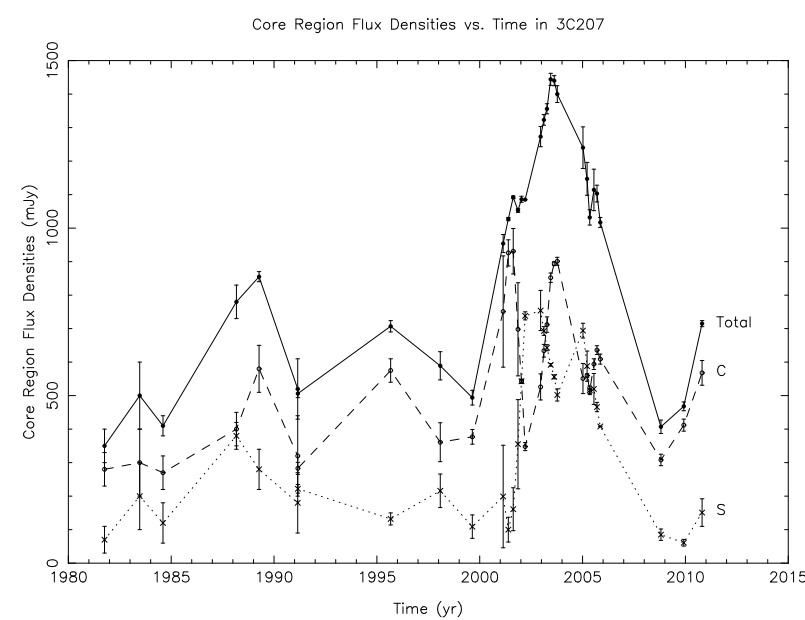

Figure 3. X-band VLBI flux densities in core region of 3C207 for true core $\mathrm{C}$, the swinging component $\mathrm{S}$, and their combined emission. The 1981.76-1991.15 data are at $10.7 \mathrm{GHz}$, and the 1991.16-2010.81 data are at $8.4 \mathrm{GHz}$.

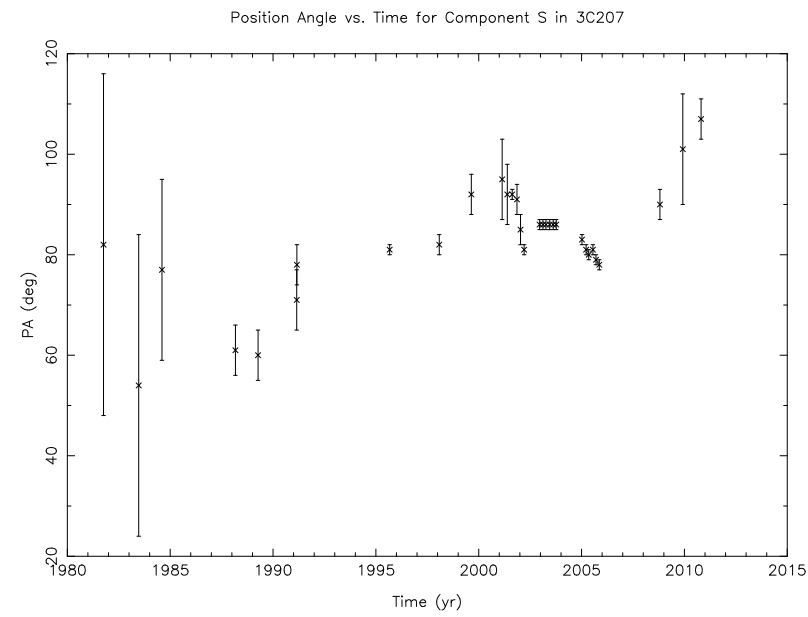

Figure 4. Position angle $P A$ vs. time for the swinging component $\mathrm{S}$ in $3 \mathrm{C} 207$.

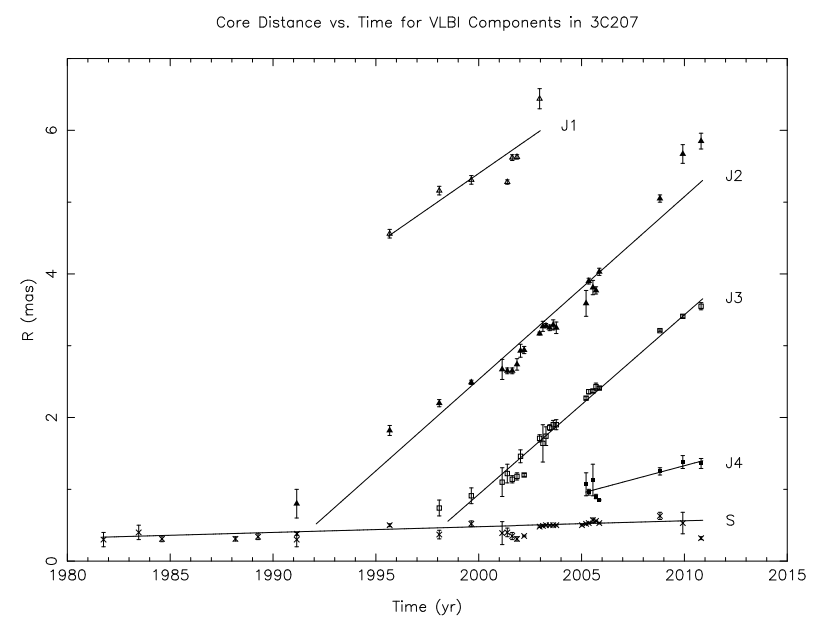

Figure 5. Core distance $R$ vs. time for the swinging component $\mathrm{S}$ and the jet components $\mathrm{J} 1, \mathrm{~J} 2, \mathrm{~J} 3$, and $\mathrm{J} 4$ in 3C207. 


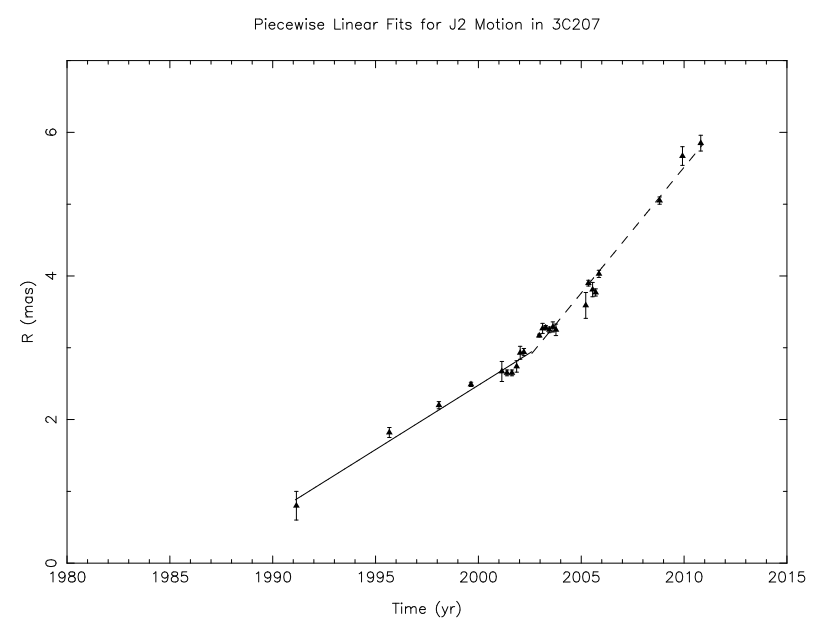

Figure 6. Core distance $R$ vs. time for jet component $\mathrm{J} 2$ in 3C207, with separate linear fits before and after epoch 2002.5, yielding superluminal speeds of $7 c$ and $14 c$, respectively.

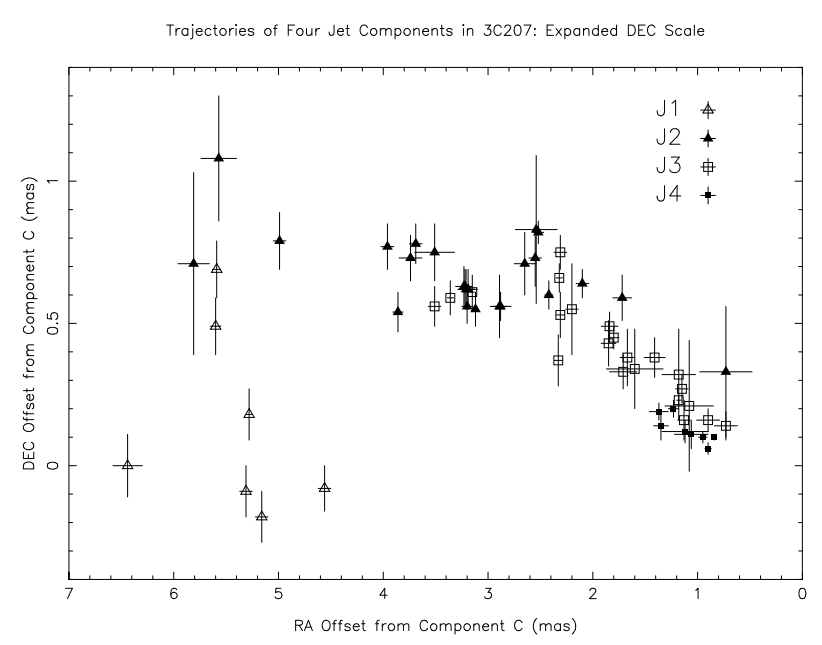

Figure 7. Trajectories on the sky for the jet components J1, J2, $\mathrm{J} 3$, and $\mathrm{J} 4$ in $3 \mathrm{C} 207$. The true core $\mathrm{C}$ is at $(0,0)$, and note the expanded DEC scale for clarity.

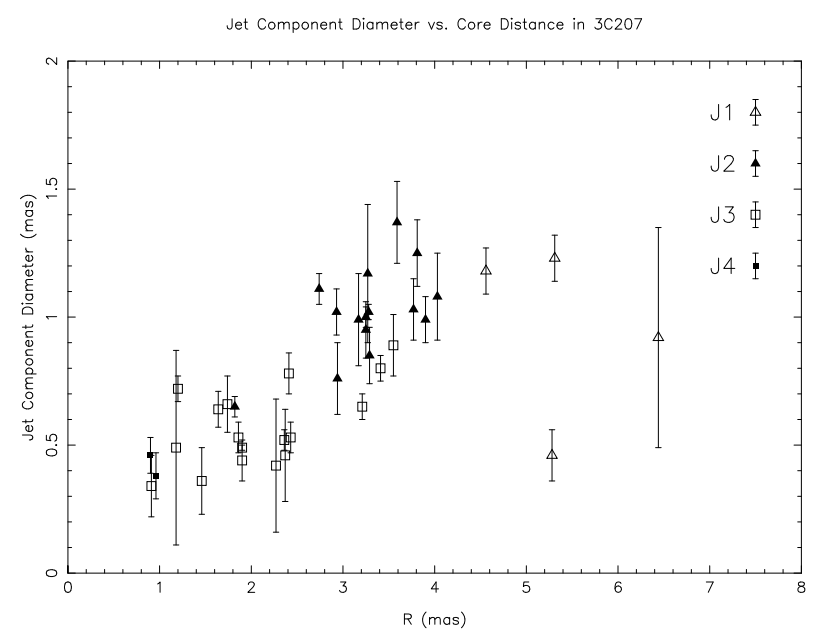

Figure 8. Jet component diameter vs. core distance $R$ in 3C207. The diameter is the FWHM of a single Gaussian component.
$(0,0)$, and note the expanded DEC offset scale for clarity. The $P A$ values of the jet knots span $\sim 25^{\circ}$, with a clear downward trend vs. time for the inner paths of $\mathrm{J} 2$, $\mathrm{J} 3$, and $\mathrm{J} 4$. There seems to be a recollimation zone that redirects the flow toward $P A \sim 90^{\circ}$ at 2-3 mas from the core - notice J2 and J3. Finally, we see that individual jet components expand until reaching the recollimation zone (Fig. 8); epochs when components had a double substructure are not included.

A working model that might explain much of the rich VLBI phenomenology in 3C207 involves a quasi-regular motion (perhaps precession) of the swinging component $\mathrm{S}$ around the axis of a cone. Deprojection of the observed $P A$ range for $\mathrm{S}$ based on the superluminal jet speeds suggests a cone with true half-angle of $\sim 3^{\circ}-4^{\circ}$. The jet components follow straight paths along this cone (so there is no evidence for helical motion related to magnetic fields). It is interesting that $\mathbf{J} 4$, with the slowest motion, is associated with the most powerful - and therefore perhaps most highy beamed - outburst in 2003 . This could be explained by motion along the cone at a very small angle to our line-of-sight. Beyond 2-3 mas, there is a narrowing of the opening angle in a recollimation zone, which accounts for the redirection of $\mathrm{J} 2$ and $\mathrm{J} 3$, and seems to be associated with their apparent acceleration and possible deceleration, respectively. While bending of the jet must play some role for both components, it is difficult to produce an increase from $7 c$ to $14 c$ in $\mathrm{J} 2$ without intrinsic acceleration.

\section{The Other Five LDQs}

The VLBI cores in the other five LDQs range from $\sim 50$ $\mathrm{mJy}$ to $\sim 500 \mathrm{mJy}$, and do not display strong variability like 3 C207. Their jets are likely oriented at larger angles to our line-of-sight. Although they display milder effects than 3 C207, they do show some similar qualitative behaviors.

\section{$3.13 \mathrm{C} 263$}

The best example is 3C263, with a VLBI core strength of $\sim 100 \mathrm{mJy}$. It has a typical core-jet structure (Fig. 9). The four jet components $\mathrm{J} 1, \mathrm{~J} 2, \mathrm{~J} 3$, and $\mathrm{J} 4$ show a pattern in which component speeds are faster further from the core: from innermost (J4) to outermost (J1), the apparent superluminal speeds are $\sim 3 c, \sim 4 c, \sim 5 c$, and $\sim 7 c$, respectively [8]. A plot of jet component $P A$ vs. $R$ shows that within $\sim 1$ mas of the core, it is clear that the components $\mathrm{J} 2, \mathrm{~J} 3$, $\mathrm{J} 4$, and the new $\mathrm{J} 5$ have emerged at successively larger $P A$, from $\sim 110^{\circ}$ to $\sim 120^{\circ}$ (Fig. 10). So while no single component is seen to accelerate as in $3 \mathrm{C} 207$, the components do move faster further from the core. And while there is no swinging component as in $3 \mathrm{C} 207$, there is a trend over time for successive components to emerge at larger $P A$.

\subsection{Additional LDQ Results}

We observe two jet components in 3C208 with superluminal speeds of $\sim 4 c$, and three in 3C249.1 with speeds of $\sim 3 c$. The core and the two jet knots in 3C208 are not 


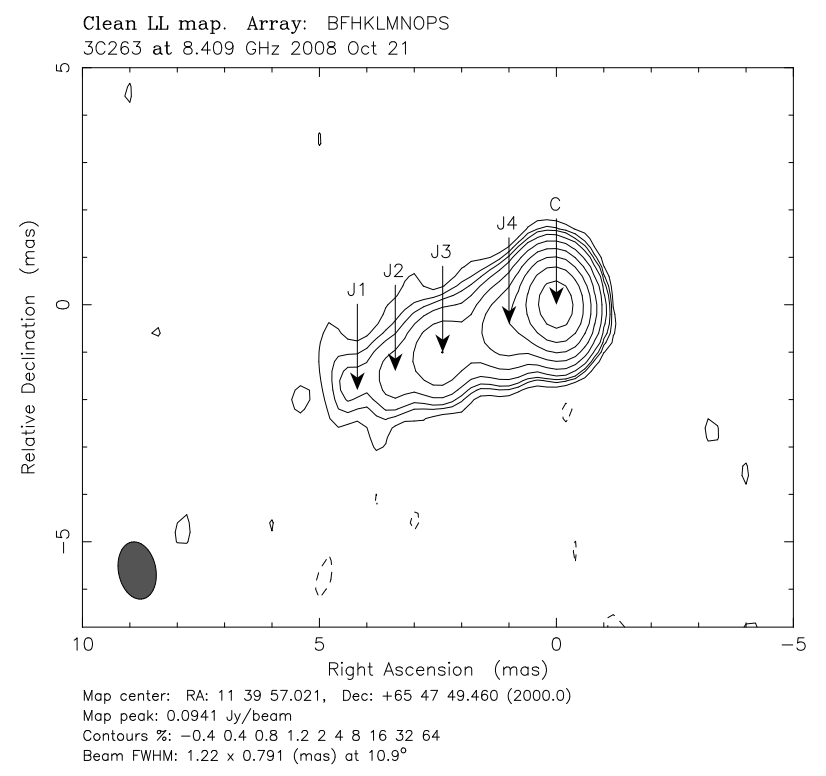

Figure 9. 8.4 GHz VLBA image of 3C263 jet at epoch 2008.81 . The core $\mathrm{C}$ and the jet components $\mathrm{J} 1, \mathrm{~J} 2$, J3, and $\mathrm{J} 4$ are labeled.

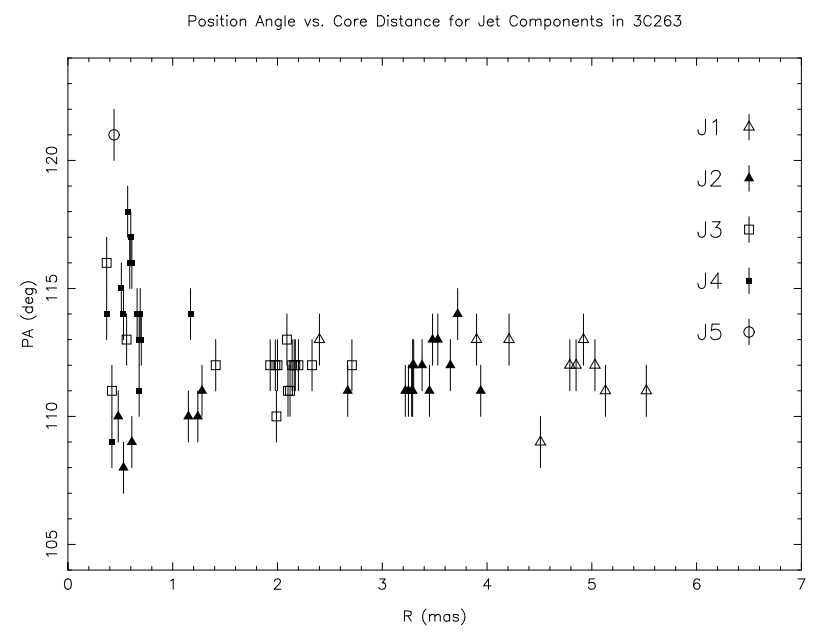

Figure 10. Position angle $P A$ vs. core distance $R$ for the jet components J1, J2, J3, J4, and the newly-emerging J5 in 3C263.

collinear. Each source has an inner feature very close to the core which we had identified as a slow-moving jet knot in our earlier studies, but which is now probably best interpreted as a stationary feature with little or no detected radial motion. The $P A$ values of the stationary features show some hints of variations, but further analysis and obsevations are needed to confirm this. Next, 3C212 has been a surprisingly quiet source despite its $\sim 150 \mathrm{mJy}$ VLBI core, with only one clear jet knot traveling at $\sim 2 c$. Finally, preliminary indications are that the inner jet knots in 3C245 range in speed between $\sim 5 c$ and $\sim 10 c$; its outer, straight $\sim 40$-mas jet traces back to a point $\sim 1-2$ mas north of the core, indicative of significant bending near the core.

\section{Conclusions and Future Work}

After thirty years, we have a working model for 3C207 in which jet components are ejected at different angles around a cone, with the flow recollimating at 2-3 mas (of order $\sim 100 \mathrm{pc}$ deprojected distance) downstream. But the jet in 3C207 is most likely aimed closest to our line-ofsight. So the question remains: How well does our model apply to the other LDQs in our sample, taking larger orientation angles, beaming, and projection effects into account? Other sources have evidence of swinging innermost components, but projection effects - and thus observed $P A$ variations - are smaller. Good determinations of the amplitude of $P A$ variations in several objects would help to constrain the intrinsic cone angle. Other sources have evidence of acceleration. But we still need to confirm trends observed thus far, make clear the circumstances in which acceleration/deceleration occurs, and determine if it is explained best by jet bending or intrinsic acceleration. Other sources show bending or misaligned jet features. But it is not clear yet when this may be associated with a recollimation zone. To address these questions, we will apply our 3C207 analysis methods to the other LDQs, and continue our VLBA monitoring.

\section{Acknowledgements}

NRAO is a facility of the NSF operated under cooperative agreement by Associated Universities, Inc. We thank the NSF RUI and AAS Small Research Grant programs. Contributors to this work include C. Aars, D. Homan, C. Newton, A. Readhead, and R. Vermeulen, and Trinity students E. Barth, T. Benavides, P. Beyer, L. Cross, E. Danielson, S. Escobedo, H. Ibaroudene, G. Jones, J. Linick, E. Phifer, N. Polito, P. Rajbhandary, B. Sadler, C. Walker, A. Webb, N. Wing, and L. Yu. N. Pape of Trinity's ITS Center maintains our astronomy workstations.

\section{References}

[1] D.H. Hough, A.C.S. Readhead, AJ 98, 1208 (1989)

[2] T.J. Pearson, A.C.S. Readhead, ApJ 328, 114 (1988)

[3] M.L. Lister, D.C. Homan, AJ 130, 1389 (2005)

[4] R.A. Laing, J.M. Riley, M.S. Longair, MNRAS 204, 151 (1983)

[5] D.H. Hough, R.C. Vermeulen, A.C.S. Readhead, L.L. Cross, E.L. Barth, L.H. Yu, P.J. Beyer, E.M. Phifer, AJ 123, 1258 (2002)

[6] E.L. Danielson, C. Smith, C. Aars, D. Hough, BAAS 39, 94 (2007)

[7] D.H. Hough, Extragalactic Jets: Theory and Observation from Radio to Gamma Ray (Astronomical Society of the Pacific, San Francisco, CA, USA, 2008) 274

[8] N. Polito, D. Hough, BAAS 42, 377 (2010) 